\title{
Modelamiento y Simulación de Redes de Transporte Urbano: Seis Casos en Lima
}

\section{Modeling and Simulation of Urban Transport Networks: Six Cases in Lima}

\author{
Jesús A. Córdova Serrano ${ }^{1}$, Sheila P. Campos Briceño ${ }^{1}$, Rosa Delgadillo ${ }^{1}$, David Mauricio ${ }^{1, a}$ \\ ${ }^{1}$ Universidad Nacional Mayor de San Marcos, Facultad de Ingeniería de Sistemas e Informática. Lima, Perú \\ admauricios@unmsm.edu.pe
}

\begin{abstract}
Resumen
Los estudios de las redes de transporte urbano en muchas ciudades no están bien enfocados, pues no contemplan herramientas para su planificación y control, haciendo que las decisiones para solucionar los múltiples problemas respecto a la congestión vehicular sean caros y no adecuadas, generando malestar en los usuarios, y muchas veces agrava los problemas, pues no se evalúa su impacto. Una alternativa a ello son los simuladores de tráfico, que en la actualidad existen en variedad, sin embargo, no son de fácil acceso, se requiere un estudio formal y una relación con el centro de investigación que lo provee, sus reportes personalizados son limitados y obedecen a otra realidad. En el presente trabajo se modela, simula seis casos frecuentes de redes de transporte urbano en Lima: Intersección, Ovalo, Unión, By-Pass, Trébol y T; mediante el uso de Arena (simulador de propósito general), técnicas estadísticas y de simulación conocidas. Se validó el modelo T con un nivel de confianza promedio del 95\%, además, se pudo obtener información personalizada para la toma de decisión.
\end{abstract}

Palabras clave: Modelos; red de transporte; simulación; congestión vehicular.

\begin{abstract}
Studies of urban transport networks in many cities are not well focused, since they do not include tools for their planning and control, making decisions to solve the multiple problems regarding traffic congestion expensive and not adequate, generating discomfort in the users, and many times it aggravates the problems, since its impact is not evaluated. Currently exist a variety of simulators that are helpful for traffic simulation and online monitoring. An alternative to this are traffic simulators, which currently exist in variety, however, they are not easily accessible, a formal study and a relationship with the research center that provides it is required, their personalized reports are limited and they comply to another reality. In the present work, six frequent cases of urban transport networks in Lima are modeled, simulated and validated: intersection, oval, union, by-pass, clover and T; by using Arena (general-purpose simulator), known statistical and simulation techniques. The T model was validated with an average confidence level of $95 \%$, in addition, personalized information could be obtained for decision making.
\end{abstract}

Keywords: Model; transport network; simulation; traffic congestion.

Correspondencia:

Dirección: Dirección: Universidad Nacional Mayor de San Marcos, Facultad de Ingeniería de Sistemas e Informática. Calle Germán Amézaga N 375 , Ciudad Lima 1.

Recibido 25/10/2020 - Aceptado 19/12/2020 - Publicado: 31/12/2020

Citar como:

Córdova, J., Campos, S., Delgadillo, R. \& Mauricio, D. (2020) Modelamiento y Simulación de Redes de Transporte Urbano: Seis Casos en Lima. Revista Peruana de Computación y Sistemas, 3(2):29-37. http://dx.doi.org/10.15381/rpcs.v3i2.19391

(c) Los autores. Este artículo es publicado por la Revista Peruana de Computación y Sistemas de la Facultad de Ingeniería de Sistemas e Informáticade la Universidad Nacional Mayor de San Marcos. Este es un artículo de acceso abierto, distribuido bajo los términos de la licencia Creative Commons Atribucion - No Comercia_Compartir Igual 4.0 Internacional. (http://creativecommons.org/licenses/by-nc-sa/4.0/) que permite el uso no comercial, distribución y reproducción en cualquier medio, siempre que la obra original sea debidamente citada. 


\section{Introducción}

Las deficiencias de transporte que enfrenta Lima se origina hace tres décadas a partir del Decreto Legislativo $\mathrm{N}^{\circ}$ 651, promulgado en Julio del año 1991; el cual declaró el libre acceso a las rutas de transporte urbano e interurbano, haciendo que las rutas y unidades crezca exponencialmente, con efectos negativos en la calidad del servicio de transporte.

El crecimiento de rutas y unidades de transporte que se viene desarrollando, tiene entre sus principales causas la alta tasa de desempleo, haciendo que muchos desempleados encuentren en el transporte público una nueva forma de subsistir. A ello, la oferta de automóviles, combis y custer usados con precios y formas de pagos muy competitivas hicieron que de forma individual o empresarial hubiera más oferta del servicio de transporte, aumentando las líneas de transporte público, hasta llegar a una sobre oferta. En consecuencia, el sector de transporte ha sufrido una sostenida degeneración de sus estructuras empresariales, las cuales son ineficientes y no incentivan la adecuada prestación del servicio, facilitando la sobreoferta, congestión, bajas velocidades de desplazamiento, alta accidentalidad, y elevados niveles de contaminación, entre otras características [1], [2]. Además, el tráfico se ve cada día más denso y lento, especialmente entre las 6:00 a 9:00 horas y 18:00 20:00 horas (horas punta) [3].

El problema de congestión de Tráfico en áreas urbanas ha estimulado mucho interés no limitado al tráfico e ingeniería de transporte. La naturaleza multidisciplinaria de tal dominio de aplicación complejo está siendo incrementalmente descubierto en diferentes campos del conocimiento, retando a los investigadores para nuevos y novedosos enfoques [2]. A primera vista, el problema obvio es que las capacidades de las redes de transporte urbanas no pueden cumplir la demanda de viaje creciente. La respuesta a este problema no es inmediata, pues requiere entendimiento de todos los factores involucrados en el sistema de transporte y sus interacciones [4]. Además, las soluciones son muy costosas e impactan fuertemente en el usuario de la red de transporte, pudiéndose transformar en nuevo problema durante o después de su implementación, por ello, se requiere que sea bien estudiadas.

Una herramienta para apoyar al desarrollo de cualquier solución al problema de la congestión del tráfico es la simulación. La simulación [5] [6] es una técnica que ayuda a la toma de decisiones, desde que esta representa un ahorro importante a la hora de concebir un proyecto. Con la simulación se puede hacer modificaciones a un sistema sin tocar el sistema real, se puede evaluar el impacto de una decisión, y ver cuál sería la opción más viable de modificación antes de implementar cualquier alternativa, lo único que se necesita son los datos de las variables involucradas en el sistema (de entrada y de salida), extraídas del sistema real, y crear modelos matemáticos, para poder simular el comportamiento del sistema real.
En la actualidad coexisten una amplia variedad de simuladores que sirven de ayuda para la simulación del tráfico y monitorización en línea. Sin embargo, estos no son de fácil acceso, se requiere un estudio formal y tener la relación con su centro de investigación; además, nos dan como resultado reportes personalizados por el usuario que son limitados u obedecen a otra realidad de contexto, y la validación de los modelos de transporte que se introducen en ellos es hecha a partir de calibración de parámetros de entrada que está a interpretación de la experiencia del usuario y además, corresponden a otras realidades.

En este trabajo se presentan el modelamiento, y simulación de redes de Transporte urbano que son frecuentes en muchas ciudades y en Lima, como: intersección, ovalo, by-pass, trébol, unión y T. Para validar los modelos se han probados con datos reales obtenidas de 6 zonas de la red urbana de Lima.

El resto de éste trabajo está organizado de la siguiente manera. En la sección 2 se presenta un breve marco teórico y revisión de literatura sobre redes de transporte y su modelamiento. Los modelos de 6 casos en Lima, su simulación mediante Arena y su validación son presentados en las secciones 3, 4 y 5 respectivamente. Finalmente, las conclusiones y trabajos futuros siguen en la sección 6.

\section{Marco Teórico y Revisión de Literatura \\ Red de Transporte}

Una red de transporte urbano se compone de una red de aceras para los peatones y una red de carreteras/ calles/jirones para los vehículos. Las aceras son la red más simple. Garantizan la movilidad a pie de las personas. Se disponen alrededor de las manzanas (o cuadras) en las zonas más próximas a los edificios con anchuras variables. En general son más anchas en las calles centrales, donde el tránsito peatonal es más intenso. $\mathrm{Cu}$ bren toda una ciudad, pero son un espacio discontinuo, y para pasar de una acera a otra es necesario cruzar la calzada por la que transitan los vehículos, para lo cual existen las señales que indican el lugar correcto donde hacerlo. Las carreteras/calles/jirones por las que circulan los vehículos son la otra red importante de las ciudades. Se extienden por casi toda una ciudad, sólo están excluidas las zonas peatonales, pero también dan acceso razonable a estas. Ambas redes tienen determinados puntos de cruce y en general, están regulados por semáforos u otro tipo de señales de tráfico.

\section{Simulación.}

La simulación es la imitación de la operación de un sistema o proceso del mundo real a través del tiempo, la simulación implica [5]: 1) la generación de una historia artificial del sistema, y 2) la observación de esa historia artificial para formular inferencias de acuerdo a las características operativas del sistema real representado. 


\section{Procesos de Poisson no estacionarios}

Con frecuencia los eventos se modelan con alguna distribución de probabilidad continua para tiempos entre eventos, y distribución discreta para el número de eventos que ocurren en un intervalo de tiempo [7]. En el caso de transporte, se experimenta variación del número de eventos en intervalos de tiempo, por ejemplo, en las horas picos (mayor tráfico) sea en la mañana tarde o noche, es decir, el proceso que gobierna las ocurrencias de evento es no estacionario. Por otro lado, no es posible ignorar estos patrones y contemplar una tasa promedio en la simulación, pues se podría obtener resultados muy inexactos, y poner en riesgo la planificación de la red de transporte. Una alternativa para esta dificultad es usar el proceso de Poisson no estacionario (NSPP, siglas en ingles). En este tipo de proceso, se requiere una función de tasa, $\lambda(t)$, que cambia con el tiempo $(t)$, que asume valores altos para tiempos $t$ cuando están sucediendo muchos eventos, y bajos cuando hay pocos.

Los eventos que ocurren en un NSPP son independientes entre sí, ocurren uno a la vez, y el número de eventos que ocurren en un intervalo de tiempo $\left[t_{1}, t_{2}\right]$ se comporta como una variable aleatoria de poisson con valor esperado dado por [5]:

$$
\Lambda\left(t_{1}, t_{2}\right)=\int_{t_{1}}^{t_{2}} \lambda(t) d t
$$

Donde el valor de $\Lambda$ es grande en intervalos de tiempo donde $\lambda(t)$ es alta y pequeña cuando $\lambda(t)$ es baja.

\section{porte}

Procedimiento de modelado de redes de Trans-

El método de modelamiento es el proceso general para un estudio de simulación, con la diferencia en el tratamiento de la data de entrada que se sigue según las recomendaciones de Rockwell Arena [8] y de Cal y Mayor [9]:

- Formulación del Problema. Se formula o plantea el problema comunicado.

- Investigación de Técnicas de Solución. Se sugiere soluciones viables y costeables.

- Investigación del sistema. Se estudia sus características de cambio, ambiente, comportamiento inesperado, caída de rendimiento e interdependencia y organización.

- Formulación del Modelo. En este paso se recopila y ajusta la data para ingresarla al modelo.

- Representación del Modelo. La geometría de la red de transporte se obtiene mediante una visita a campo, al igual que los flujos. Al tener identificados los puntos de entrada a la red, estos se etiquetan en el sentido inverso de las agujas del reloj, se pueden etiquetar con letras A, B, C, etc.; o con números 10, 20,30, etc. Luego se codifican los giros o movimientos ya identificados de cada entrada a la red de izquierda a derecha (A, A1, A2, B, B1, B2, etc. en caso de letras, y 11,12, 13, $21,22,23$, etc. en caso de números) [4], para poder contabilizar el flujo por tipo de auto, este se toma en intervalos de 15 minutos.

- Programación. Pasar el modelo a un ordenador (simulador).

- Diseño de Experimentos. Crear casos de prueba, definiendo las variables de salida.

- Experimentación. Ejecutar modelo en simulador y validar el modelo.

- Redefinición. Redefinir el modelo en caso de fallo.

- Presentación de los resultados de simulación. Presentar los resultados a los involucrados.

\section{Validación por Intervalos de Confianza.}

Para estimar la distribución de la muestra para una población que siga una distribución normal o los principios del teorema del límite central se usa el enfoque de estimación de intervalos [10], por ejemplo, para un parámetro $\mu$ no se estima un valor, sino un intervalo de la forma $L \leq \mu \leq U$, donde los valores extremos $L, U$ dependen del valor numérico del estadístico $\bar{x}$ para una muestra en particular y de la distribución de muestreo.

Partiendo de la distribución de muestreo para $\bar{x}$, es posible determinar valores de $\mathrm{L}, \mathrm{U}$ tales que se cumpla lo siguiente: $P(L \leq \mu \leq U)=1-\alpha$ Donde $0<\alpha<1$. Es decir, se puede garantizar con una probabilidad de $1-\alpha$ (Este es el nivel de confianza, que se validará luego si el modelo está bien ajustado) de que la muestra elegida contendrá el valor verdadero de $\mu$. Al intervalo resultante $L \leq \mu \leq U$ se le conoce como el intervalo de confianza del $100(1-\alpha) \%$ para el parámetro desconocido $\mu$. Los intervalos de confianza siguen la formula siguiente, que se halla a partir de una Distribución Normal tipificada:

$$
\bar{x}-z_{\alpha / 2} \sigma / \sqrt{N} \leq \mu \leq \bar{x}+z_{\alpha / 2} \sigma / \sqrt{N}
$$

Donde $\bar{x}$ es la media muestral, $\mathbf{z}_{\alpha / 2}$ es el valor extraído de la tabla normal para un nivel de significación $\boldsymbol{\alpha}$, gamma $(\boldsymbol{\sigma})$ es la desviación estándar de la población y $\mathrm{N}$ es el tamaño de la muestra. La ecuación (2) es para muestras mayores a 30, y para muestras menores a 30, el intervalo de confianza se halla de la siguiente forma, ecuación (3):

$$
\bar{x} \pm t_{n-1, \alpha / 2} \sqrt{\frac{S^{2}(n)}{n}}
$$

Donde $\bar{x}$ es la media muestral, $t_{\mathrm{n}-1, \alpha / 2}$ es el valor extraído de la tabla de la distribución t de student, $S$ es la desviación estándar de la muestra y $n$ es el tamaño de la muestra.

Para muestras grandes $(N>30)$, el valor $\sigma$ se puede estimar por $S$. 


\section{Taxonomía de Redes de Transporte}

Las redes de transporte se dividen en dos categorías [11]: redes de transporte urbano y redes de transporte interurbano. En estas últimas, no se presentan muchos problemas de congestión, ya que la mayoría de veces se encuentra fuera de la urbe de la ciudad y en general hay ausencia de tráfico, salvo en casos excepcionales. Las redes de transporte urbano son más complejos que las redes de transporte interurbano, esto porque presentan diversos factores y general se presentan con mayor frecuencia, como: densidad de vehículos (mucho mayor cuanto más importante es el núcleo urbano objeto del estudio), intersecciones (que se incrementan en los núcleos de población), incidentes (de los que podríamos hacer subdivisiones y descripciones de cada uno de ellos; cortes de calles debidos a accidentes, obras, transportes especiales, vehículos propios de una ciudad, etc..), semáforos (con la complicada gestión de las intersecciones que gobiernan)

\section{Algunos Simuladores de Transporte}

Existen diversos simuladores para redes de transporte, en la Figura 1 se muestran 20 características de los simuladores presentados en [12].

\section{Casos de Estudio}

En [13] se desarrolló un modelo de micro simulación para los servicios de bus de la ciudad de York, Reino Unido, zona turística, localizada al norte de Inglaterra usando el simulador DRACULA, con la cual, se simuló escenarios de horas picos y flujos en dirección del área residencial hacia el centro de la ciudad por la mañana y en sentido contrario por la tarde, además, la variación de tiempos entre buses y el tiempo de espera de los pasajeros.

En [14] se desarrolla un modelo de micro simulación basado en DRACULA de una sección de un corredor principal Soweto a Sunninghill, en la zona sur de la ciudad de Johannesburg, Sudafrica. El corredor en estudio tiene aproximadamente $22 \mathrm{~km}$. de longitud, consiste de 77 intersecciones, de las cuales 39 están seńalizadas. La mayoría de la ruta es de ida y vuelta dividida en 4 carriles, algunas secciones localizadas del camino tienen solo 2 carriles. Es característico la congestión severa durante horas pico, además considera los servicios de taxis, vehículos privados y transporte público, siendo esta última entre $85 \%$ y $90 \%$ de los viajes en la sección sur y entre $55 \%$ y $65 \%$ en la sección norte. El modelo permite desarrollar diversos escenarios, con lo cual se ha conseguido una marcada mejora en el tiempo de viaje en transporte público en el corredor, sin impacto negativo en otros usuarios. Además, el incremento del tráfico privado, en el futuro hará que el tiempo de viaje de esto usuarios exceda al del transporte público, proporcionando un incentivo para cambiar al transporte público.

\section{Modelamiento de casos de transporte urbano Lima, Perú}

En la presente sección se diseña 6 modelos de redes de transporte para la ciudad de Lima, y que es frecuente en las ciudades:

- Modelo Intersección. Implica un cruce semaforizado de dos avenidas, con giros permitidos variables.

\begin{tabular}{|c|c|c|c|c|c|c|c|c|c|c|c|c|c|c|c|c|c|c|c|c|c|}
\hline & 1 & 2 & 3 & 4 & 5 & 6 & 7 & 8 & 9 & 10 & 11 & 12 & 13 & 14 & 15 & 16 & 17 & 18 & 19 & 20 & 21 \\
\hline AIMSUN 2 & $\mathrm{X}$ & $\mathrm{X}$ & & $\mathrm{X}$ & & $\mathrm{X}$ & $\mathrm{X}$ & $\mathrm{X}$ & & $\mathrm{X}$ & $\mathrm{X}$ & & & $\mathrm{X}$ & & & & & & & $\mathrm{X}$ \\
\hline ANATOLL & & & & & & & & & & & & & & & $\mathrm{X}$ & & & & & & \\
\hline AUTOBAHN & $\mathrm{X}$ & $\mathrm{X}$ & & $\mathrm{X}$ & $\mathrm{X}$ & $\mathrm{X}$ & $\mathrm{X}$ & $\mathrm{X}$ & $\mathrm{X}$ & $\mathrm{X}$ & $\mathrm{X}$ & $\mathrm{X}$ & & $\mathrm{X}$ & $\mathrm{X}$ & $\mathrm{X}$ & $\mathrm{X}$ & $\mathrm{X}$ & & $\mathrm{X}$ & $\mathrm{X}$ \\
\hline CASIMIR & & $\mathrm{X}$ & & & & & & & & & & & & & & & & & & & $\mathrm{X}$ \\
\hline CORSIM & $\mathrm{X}$ & $\mathrm{X}$ & $\mathrm{X}$ & $\mathrm{X}$ & $\mathrm{X}$ & $\mathrm{X}$ & & & & & & & & & & & & & & & \\
\hline DRACULA & $\mathrm{X}$ & $\mathrm{X}$ & $\mathrm{X}$ & & & & & & & & & & & & $\mathrm{X}$ & & & & & & $\mathrm{X}$ \\
\hline FLEXSYT II & $\mathrm{X}$ & $\mathrm{X}$ & $\mathrm{X}$ & $\mathrm{X}$ & $\mathrm{X}$ & $\mathrm{x}$ & $\mathrm{X}$ & & & & & & & $\mathrm{X}$ & & & & & $\mathrm{X}$ & & $\mathrm{X}$ \\
\hline FREEVU & & & & & & & & & & & & & & & & & & & & $\mathrm{X}$ & $\mathrm{X}$ \\
\hline FRESIM & & & & $\mathrm{X}$ & $\mathrm{X}$ & $\mathrm{X}$ & & & & & & & & & & & & & & & $\mathrm{X}$ \\
\hline HUTSIM & $\mathrm{X}$ & $\mathrm{X}$ & $\mathrm{X}$ & $\mathrm{X}$ & & & & $\mathrm{X}$ & & $\mathrm{X}$ & & & & $\mathrm{X}$ & & $\mathrm{X}$ & & $\mathrm{X}$ & $\mathrm{X}$ & $\mathrm{X}$ & $\mathrm{X}$ \\
\hline INTEGRATION & $\mathrm{X}$ & $\mathrm{X}$ & $\mathrm{X}$ & $\mathrm{X}$ & $\mathrm{X}$ & $\mathrm{X}$ & & $\mathrm{X}$ & & $\mathrm{X}$ & $\mathrm{X}$ & & $\mathrm{X}$ & $\mathrm{X}$ & $\mathrm{X}$ & & & & & $\mathrm{X}$ & $\mathrm{X}$ \\
\hline MELROSE & $\mathrm{X}$ & $\mathrm{X}$ & & $\mathrm{X}$ & $\mathrm{X}$ & & $\mathrm{X}$ & & & $\mathrm{X}$ & $\mathrm{X}$ & & & $\mathrm{X}$ & $\mathrm{X}$ & $\mathrm{X}$ & $\mathrm{X}$ & $\mathrm{X}$ & & $\mathrm{X}$ & $\mathrm{X}$ \\
\hline MICROSIM & & $\mathrm{X}$ & & $\mathrm{X}$ & & & & & & $\mathrm{X}$ & $\mathrm{X}$ & & & & & & & & & & \\
\hline MICSTRAN & $\mathrm{X}$ & $\mathrm{X}$ & $\mathrm{X}$ & $\mathrm{X}$ & & & $\mathrm{X}$ & & & $\mathrm{X}$ & $\mathrm{X}$ & $\mathrm{X}$ & & & $\mathrm{X}$ & & & & & & $\mathrm{X}$ \\
\hline MITSIM & $\mathrm{X}$ & $\mathrm{X}$ & & $\mathrm{X}$ & $\mathrm{X}$ & $\mathrm{X}$ & & $\mathrm{X}$ & & $\mathrm{X}$ & $\mathrm{X}$ & & & $\mathrm{X}$ & & & & & & $\mathrm{X}$ & $\mathrm{X}$ \\
\hline MIXIC & & & & & $\mathrm{X}$ & & & & & & & & & & & $\mathrm{X}$ & $\mathrm{X}$ & $\mathrm{X}$ & & & \\
\hline NEMIS & $\mathrm{X}$ & $\mathrm{X}$ & $\mathrm{X}$ & & & $\mathrm{X}$ & $\mathrm{X}$ & $\mathrm{X}$ & & $\mathrm{X}$ & $\mathrm{X}$ & & & & & $\mathrm{X}$ & & & & $\mathrm{X}$ & $\mathrm{X}$ \\
\hline NETSIM & $\mathrm{X}$ & $\mathrm{X}$ & $\mathrm{X}$ & & & $\mathrm{X}$ & & & & & & & & & & & & & & & $\mathrm{X}$ \\
\hline PADSIM & $\mathrm{X}$ & $\mathrm{X}$ & & & & & $\mathrm{X}$ & & & & $\mathrm{X}$ & & & & & $\mathrm{X}$ & & & & & $\mathrm{X}$ \\
\hline PARAMICS & $\mathrm{X}$ & $\mathrm{X}$ & & $\mathrm{X}$ & $\mathrm{X}$ & $\mathrm{x}$ & $\mathrm{X}$ & $\mathrm{X}$ & $\mathrm{X}$ & $\mathrm{X}$ & $\mathrm{X}$ & & & $\mathrm{X}$ & $\mathrm{X}$ & & $\mathrm{X}$ & & & $\mathrm{X}$ & $\mathrm{X}$ \\
\hline PHAROS & $\mathrm{X}$ & & & & & & & & & & & & & & & & & & & & \\
\hline PLANSIM- $T$ & $\mathrm{X}$ & $\mathrm{X}$ & $\mathrm{X}$ & $\mathrm{X}$ & & & $\mathrm{X}$ & $\mathrm{X}$ & $\mathrm{X}$ & $\mathrm{X}$ & $\mathrm{X}$ & $\mathrm{X}$ & & & $\mathrm{X}$ & & $\mathrm{X}$ & & & $\mathrm{X}$ & \\
\hline SHIVA & & & & & & & & & & & & & & & & $\mathrm{X}$ & $\mathrm{X}$ & $\mathrm{X}$ & & & $\mathrm{X}$ \\
\hline SIGSIM & $\mathrm{X}$ & $\mathrm{X}$ & $\mathrm{X}$ & $\mathrm{X}$ & $\mathrm{X}$ & $\mathrm{X}$ & & & & & & & $\mathrm{X}$ & & & $\mathrm{X}$ & & $\mathrm{X}$ & & $\mathrm{X}$ & $\mathrm{X}$ \\
\hline SIMDAC & & & & & & & & & & & & & & & & $\mathrm{X}$ & & & & & \\
\hline SIMNET & $\mathrm{X}$ & $\mathrm{X}$ & $\mathrm{X}$ & $\mathrm{X}$ & & $\mathrm{X}$ & & $\mathrm{X}$ & & $\mathrm{X}$ & $\mathrm{X}$ & $\mathrm{X}$ & & & & $\mathrm{X}$ & & & & & $\mathrm{X}$ \\
\hline SISTM & & & & $\mathrm{X}$ & $\mathrm{X}$ & & & $\mathrm{X}$ & & $\mathrm{X}$ & & & & & & & & & & & $\mathrm{X}$ \\
\hline SITRA-B+ & $\mathrm{X}$ & $\mathrm{X}$ & $\mathrm{X}$ & & & $\mathrm{X}$ & & & & $\mathrm{X}$ & $\mathrm{X}$ & $\mathrm{X}$ & & & & & & & & $\mathrm{X}$ & $\mathrm{X}$ \\
\hline SITRAS & $\mathrm{X}$ & $\mathrm{X}$ & & & & $\mathrm{X}$ & & & & $\mathrm{X}$ & $\mathrm{X}$ & & & & & & & & & & $\mathrm{X}$ \\
\hline THOREAU & $\mathrm{X}$ & $\mathrm{X}$ & & $\mathrm{X}$ & & & & $\mathrm{X}$ & & $\mathrm{X}$ & $\mathrm{X}$ & & & & & & & & & $\mathrm{X}$ & $\mathrm{X}$ \\
\hline VISSIM & $\mathrm{X}$ & $\mathrm{X}$ & $\mathrm{X}$ & $\mathrm{X}$ & $\mathrm{X}$ & & & & & & & & $\mathrm{X}$ & & & & & & $\mathrm{X}$ & $\mathrm{X}$ & $\mathrm{X}$ \\
\hline
\end{tabular}

Leyenda (características y capacidades)

1. Señales de tráfico coordinadas.

2. Señales de tráfico adaptables.

3. Prioridad a vehículos de transporte público.

4. Entrada de rampa.

5. Control de flujo de carretera.

6. Gestión de incidentes.

7. Control de acceso a zona.

8. Señales de mensaje variables.

9. Información de tráfico regional.

10. Guía de rutas estáticas.

11. Guía de rutas dinámicas.

12. Guía parking.

13. Información de transporte público.

14. Plazas de peaje y débito automáticas.

15. Tasación de congestión.

16. Estabilizador de velocidad automático.

17. Sistema de carreteras automatizado.

18. Vehículos autónomos.

19. Soporte para peatones y ciclistas.

20. Probar vehículos.

21. Detectores de vehículos.

Figura 1. Características y capacidades de simuladores de transportes (desde [12]) 
Caso de Estudio: Av. Central con Av. Cesar Vallejo, Villa El Salvador.

- Modelo Ovalo. Implica un ovalo de más de un carril central con varias entradas y salidas del mismo, las entradas se comportan como salida y viceversa. Caso de Estudio: Plaza Ramón Castilla, Cercado de Lima.

- Modelo Unión. Implica una carretera, una rampa de entrada a la misma, un carril de aceleración de cierta magnitud, donde los carros se unen a la carretera bajo ciertas situaciones antes que se termine el carril de aceleración, necesaria para mantener la velocidad en la carretera. Caso de Estudio: Av. Riva Agüero con Av. Nicolás Ayllon, Ate.

- Modelo By-Pass. Implica un cruce de dos avenidas, el flujo de tráfico en una de ellas es más elevado que en la otra, de modo que no hay cruce físico. Existencia de vías auxiliares. Caso de Estudio: Av. Oscar R. Benavides con Av. Universitaria, Cercado de Lima.

- Modelo Trébol. Implica un cruce tipo By-Pass pero semejante a un trébol, con rampas para subir y bajar del puente. Caso de Estudio: Trébol de Javier Prado, San Borja.

- Modelo T. Implica un cruce en T. Caso de Estudio: Av. Veronesse con Av. Morelli, San Borja.

Se procederá a modelar los 6 casos de estudio siguiendo el procedimiento de modelado de redes de transporte presentado en la sección 2. El estudio fue realizado con datos reales de la ciudad de Lima y corresponden al año 2012, detalle de ellos se encuentran en [15].

\section{Investigación del sistema}

- La red de transporte no va a cambiar durante el curso del estudio.

- Las variables de entradas son el flujo vehicular por tipo de vehículo y los intervalos de tiempo.

- Los Peatones, vehículos de emergencia y ciclistas están excluidos de la definición del sistema debido a su efecto negativo en el estado del mismo.

- La performance del sistema no se deteriora en el tiempo.

\section{Formulación del Modelo}

- Están hechas todas las suposiciones abstrayendo la red de transporte para cumplir los objetivos de estudio.

- La data recolectada ha sido provista por la Secretaria Técnica de Transporte de Lima y Callao [16] y La Gerencia de transporte Urbano [17].

- La data recolectada sigue una distribución de Poisson no estacionaria.

\section{Representación del Modelo}

La geometría de los 6 casos de estudios se muestra en la figura 2, estas fueron realizadas con la herramienta Arena [8] en su versión estudiantil.

\section{Programación}

Se usó la herramienta Arena [8], dado que permite la integración con Lenguajes de Programación y la emisión de reportes personalizados, además, porque se integra con otras herramientas complementarias para el tratamiento y validación de la data.

\section{Diseño de Experimentos}

Se especifica el método de replicación para análisis estadístico de la salida de la simulación. Las variables de salida consideradas son: los flujos vehiculares correspondientes a la hora punta del total del flujo tomado.

\section{Experimentación}

Se experimenta con el modelo construido en ARENA y se hallan los intervalos de confianza, esto se explica en la siguiente sección.

\section{Proceso de Simulación de Tráfico en Arena}

Con la geometría identificada, procesamos la data de entrada, obtenida de los estudios de transporte. La data se agrupa por tipo de vehículo y por movimiento e intervalos de 15 minutos, como se aprecia en la figura 3.

A seguir se modelo en Arena cada caso. El modelo en Arena fue diseñado para cada caso y esta descrito en [15], en la Figura 4 se muestra el caso del modelo T, en donde se observa dos estructuras para la red de transporte: la Entrada a Red y Línea de Parada, que representa un punto de entrada a la red hasta la línea de parada indicada por una señal de tránsito; y la Salida de la Red, que representa la entrada a una vía de salida de la red de Transporte.

Seguidamente, obtenemos la animación del modelo como se aprecia en la Figura 5.

\section{Validación de modelos de Redes de Transporte}

El modelo se validó mediante la técnica de los intervalos de confianza [10] y se hizo para las variables que se muestran en la Figura 6, obteniendo un nivel de confianza en promedio del 90 al 99\%. Las variables fueron validadas contra el promedio de cada variable simulada y real. La confianza será más alta conforme el número de réplicas de simulación incremente.

La Figura 6 muestra en las columnas las variables correspondientes a la hora punta, el porcentaje de confianza es calculado en base a la cantidad de intervalos que contienen a la media real o simulada de cada variable entre el número total de intervalos hallados. 


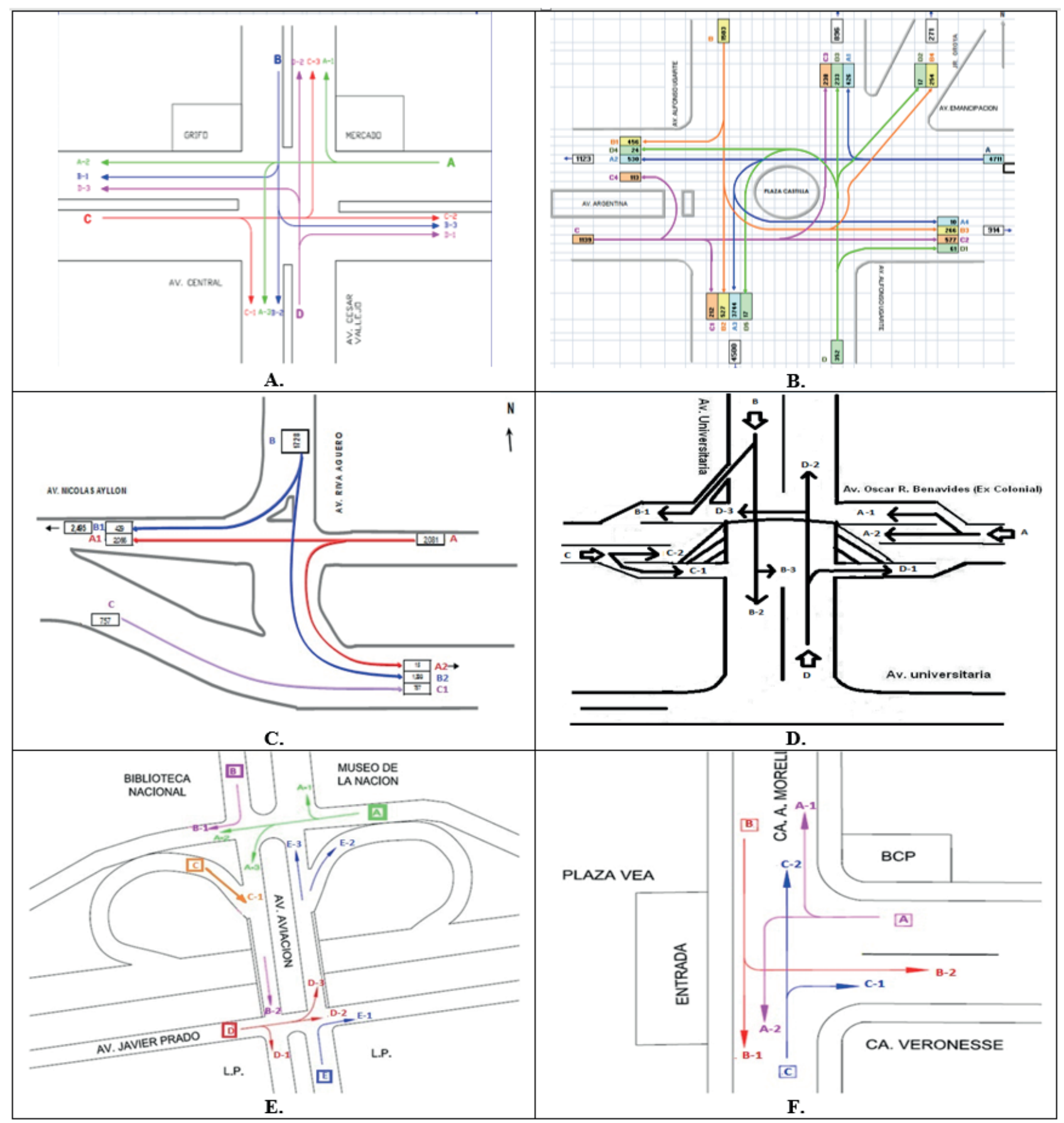

Figura 2. Geometría de los casos de estudio: A) Intersección; B) Ovalo; C) Unión; D) By-pass; E) Trébol; F) T 


\begin{tabular}{|c|c|c|c|c|c|c|c|c|c|c|c|c|c|c|c|c|c|c|c|c|c|c|c|c|}
\hline \multirow[t]{2}{*}{ MORA } & \multicolumn{6}{|c|}{ AUTO } & \multicolumn{6}{|c|}{ Bicicletas } & \multicolumn{6}{|c|}{ MOTOCICLETAS - MOTO-tazI } & \multicolumn{6}{|c|}{ camion } \\
\hline & A-1 & A-2 & B-1 & B-2 & c-1 & $c-2$ & A-1 & $A-2$ & B-1 & B-2 & c-1 & $c-2$ & A-1 & $A-2$ & B-1 & $B-2$ & c-1 & c-2 & A-1 & A-2 & B-1 & B-2 & c-1 & c-2 \\
\hline $7: 00-7: 15$ & 0 & 0 & 11 & 4 & 14 & 17 & 0 & 0 & 1 & 0 & 0 & 2 & 1 & 0 & 1 & 0 & 0 & 0 & 0 & 0 & 0 & 0 & 1 & 0 \\
\hline $7: 15-7: 30$ & 0 & 0 & 15 & 5 & 8 & 26 & 0 & 0 & 0 & 0 & 0 & 1 & 1 & 0 & 0 & 0 & 0 & 0 & 0 & 0 & 0 & 0 & 0 & 0 \\
\hline $7: 30-7: 45$ & 0 & 0 & 26 & 8 & 16 & 23 & 0 & 0 & 0 & 0 & 0 & 0 & 2 & 0 & 0 & 0 & 0 & 0 & 0 & 0 & 0 & 0 & 1 & 0 \\
\hline $7: 45-8: 00$ & 0 & 0 & 19 & 16 & 42 & 13 & 0 & 0 & 0 & 0 & 2 & 1 & 0 & 0 & 0 & 0 & 0 & 0 & 0 & 0 & 0 & 0 & 0 & 0 \\
\hline 8:00 - 8:15 & 15 & 17 & 32 & 18 & 72 & 15 & 0 & 0 & 1 & 0 & 0 & 0 & 1 & 2 & 1 & 0 & 1 & 3 & 1 & 0 & 0 & 0 & 0 & 0 \\
\hline $8: 15-8: 30$ & 18 & 27 & 20 & 10 & 79 & 10 & 0 & 0 & 0 & 0 & 1 & 0 & 0 & 0 & 1 & 0 & 0 & 0 & 0 & 0 & 0 & 0 & 6 & 0 \\
\hline $8: 30-8: 45$ & 33 & 19 & 36 & 11 & 105 & 5 & 1 & 0 & 1 & 0 & 0 & 0 & 1 & 1 & 2 & 0 & 6 & 2 & 0 & 0 & 1 & 0 & 0 & 0 \\
\hline $8: 45-9: 00$ & 16 & 28 & 28 & 7 & 114 & 11 & 0 & 1 & 0 & 0 & 0 & 0 & 0 & 2 & 0 & 0 & 2 & 4 & 0 & 0 & 0 & 0 & 1 & 0 \\
\hline 17:00 - 17:15 & 30 & 23 & 41 & 19 & 68 & 16 & 0 & 0 & 1 & 1 & 1 & 0 & 2 & 0 & 3 & 0 & 1 & 0 & 0 & 0 & 1 & 0 & 3 & 0 \\
\hline $17: 15-17: 30$ & 26 & 25 & 51 & 24 & 70 & 19 & 0 & 0 & 1 & 0 & 0 & 0 & 1 & 1 & 2 & 1 & 2 & 1 & 0 & 0 & 3 & 1 & 1 & 0 \\
\hline $17: 30-17: 45$ & 32 & 27 & 62 & 20 & 83 & 19 & 0 & 0 & 1 & 0 & 0 & 0 & 1 & 3 & 4 & 1 & 1 & 0 & 0 & 0 & 0 & 0 & 1 & 0 \\
\hline 17:45 - 18:00 & 40 & 27 & 63 & 32 & 73 & 31 & 0 & 0 & 1 & 0 & 1 & 1 & 2 & 0 & 1 & 0 & 1 & 0 & 1 & 0 & 1 & 0 & 2 & 0 \\
\hline $18: 00-18: 15$ & 36 & 23 & 56 & 18 & 53 & 25 & 0 & 0 & 0 & 0 & 1 & 2 & 1 & 1 & 2 & 1 & 4 & 2 & 0 & 0 & 1 & 0 & 0 & 0 \\
\hline $18: 15-18: 30$ & 37 & 21 & 68 & 16 & 71 & 34 & 1 & 0 & 0 & 0 & 0 & 0 & 2 & 0 & 2 & 2 & 1 & 0 & 0 & 2 & 0 & 0 & 6 & 2 \\
\hline $18: 30-18: 45$ & 40 & 30 & 56 & 12 & 65 & 28 & 1 & 0 & 0 & 0 & 1 & 0 & 1 & 0 & 0 & 0 & 2 & 0 & 1 & 0 & 1 & 0 & 0 & 0 \\
\hline $18: 45-19: 00$ & 40 & 23 & 33 & 12 & 60 & 10 & 0 & 0 & 0 & 0 & 0 & 0 & 0 & 1 & 1 & 1 & 0 & 0 & 0 & 0 & 1 & 1 & 0 & 0 \\
\hline
\end{tabular}

\begin{tabular}{|c|c|c|c|c|c|c|c|c|c|c|c|c|c|c|c|c|c|c|c|c|c|c|c|c|}
\hline \multirow[t]{2}{*}{ HORA } & \multicolumn{6}{|c|}{ сонві } & \multicolumn{6}{|c|}{ MICROBUS } & \multicolumn{6}{|c|}{ онmiвus } & \multicolumn{6}{|c|}{ otRos } \\
\hline & A-1 & $A-2$ & B-1 & B-2 & c-1 & c-2 & A-1 & $A-2$ & B-1 & B-2 & c-1 & c-2 & A-1 & A-2 & B-1 & B-2 & c-1 & c-2 & A-1 & A-2 & B-1 & B-2 & c-1 & e-2 \\
\hline $7: 00$ - 7:15 & 0 & 0 & 0 & 1 & 0 & 1 & 0 & 0 & 0 & 0 & 0 & 0 & 0 & 0 & 0 & 0 & 0 & 0 & 0 & 0 & 0 & 0 & 0 & 0 \\
\hline 7:15- 7:30 & 0 & 0 & 0 & 0 & 0 & 1 & 0 & 0 & 0 & 0 & 0 & 0 & 0 & 0 & 0 & 0 & 0 & 0 & 0 & 0 & 0 & 0 & 0 & 0 \\
\hline $7: 30-7: 45$ & 0 & 0 & 1 & 0 & 0 & 0 & 0 & 0 & 0 & 0 & 0 & 0 & 0 & 0 & 0 & 0 & 0 & 0 & 0 & 0 & 0 & 0 & 0 & 0 \\
\hline 7:45 - 8:00 & 0 & 0 & 0 & 0 & 0 & 1 & 0 & 0 & 0 & 0 & 0 & 1 & 0 & 0 & 0 & 0 & 0 & 0 & 0 & 0 & 2 & 0 & 0 & 0 \\
\hline $8: 00-8: 15$ & 0 & 0 & 0 & 0 & 1 & 1 & 0 & 0 & 0 & 0 & 0 & 0 & 0 & 0 & 0 & 0 & 0 & 0 & 0 & 0 & 3 & 1 & 0 & 1 \\
\hline $8: 15-8: 30$ & 0 & 0 & 1 & 0 & 0 & 0 & 0 & 0 & 0 & 0 & 0 & 0 & 0 & 0 & 0 & 0 & 0 & 0 & 0 & 0 & 9 & 1 & 0 & 0 \\
\hline $8: 30-8: 45$ & 0 & 0 & 1 & 0 & 1 & 1 & 0 & 0 & 0 & 0 & 0 & 0 & 0 & 0 & 0 & 0 & 0 & 0 & 0 & 0 & 9 & 0 & 0 & 0 \\
\hline $8: 45$ - 9:00 & 0 & 0 & 0 & 0 & 0 & 2 & 0 & 0 & 0 & 0 & 0 & 0 & 0 & 0 & 0 & 0 & 0 & 0 & 0 & 0 & 20 & 8 & 0 & 0 \\
\hline $17: 00-17: 15$ & 0 & 0 & 0 & 0 & 2 & 0 & 0 & 0 & 0 & 0 & 0 & 0 & 0 & 0 & 0 & 0 & 0 & 0 & 0 & 0 & 21 & 21 & 0 & 0 \\
\hline $17: 15-17: 30$ & 1 & 1 & 1 & 0 & 1 & 0 & 0 & 0 & 0 & 0 & 0 & 0 & 0 & 0 & 0 & 0 & 0 & 0 & 0 & 0 & 22 & 19 & 0 & 0 \\
\hline $17: 30-17: 45$ & 0 & 0 & 1 & 0 & 3 & 1 & 0 & 0 & 0 & 0 & 0 & 0 & 0 & 0 & 0 & 0 & 0 & 0 & 0 & 0 & 22 & 20 & 0 & 0 \\
\hline $17: 45-18: 00$ & 0 & 0 & 0 & 1 & 1 & 0 & 0 & 0 & 0 & 0 & 0 & 0 & 0 & 0 & 0 & 0 & 0 & 0 & 0 & 0 & 19 & 20 & 0 & 0 \\
\hline 18:00 - 18:15 & 0 & 1 & 0 & 1 & 1 & 1 & 0 & 0 & 0 & 0 & 0 & 0 & 0 & 0 & 0 & 0 & 0 & 0 & 0 & 0 & 20 & 17 & 0 & 0 \\
\hline $18: 15$ - 18:30 & 1 & 0 & 0 & 1 & 2 & 0 & 0 & 0 & 0 & 0 & 0 & 0 & 0 & 0 & 0 & 0 & 0 & 0 & 0 & 0 & 32 & 30 & 0 & 0 \\
\hline $18: 30-18: 45$ & 0 & 0 & 1 & 0 & 2 & 2 & 0 & 0 & 0 & 0 & 0 & 0 & 0 & 0 & 0 & 0 & 0 & 0 & 0 & 0 & 33 & 28 & 0 & 0 \\
\hline $18: 45-19: 00$ & 0 & 0 & 0 & 0 & 2 & 0 & 0 & 0 & 0 & 0 & 0 & 0 & 0 & 0 & 0 & \begin{tabular}{|l|l}
0 \\
\end{tabular} & \begin{tabular}{|l|l}
0 \\
\end{tabular} & \begin{tabular}{|l|l|}
0 & \\
\end{tabular} & 0 & \begin{tabular}{|l|l}
0 \\
\end{tabular} & \begin{tabular}{|l|l|}
21 \\
\end{tabular} & \begin{tabular}{|l|l|}
15 \\
\end{tabular} & \begin{tabular}{|l|l}
0 \\
\end{tabular} & 0 \\
\hline
\end{tabular}

Figura 3. Data de flujos de tráfico [16]

\section{Entrada a Red y linea de Parada द)}

\section{Salida de Red}<smiles>C1CC2CCC(C1)C2</smiles>
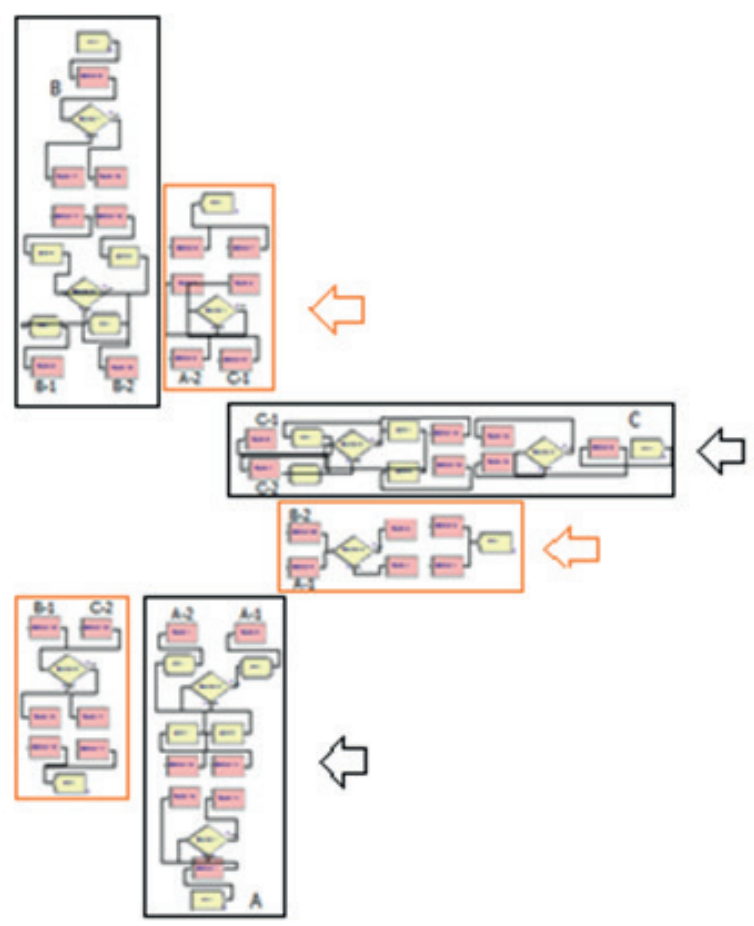
ret

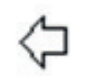

Figura 4. Modelo T en Arena 


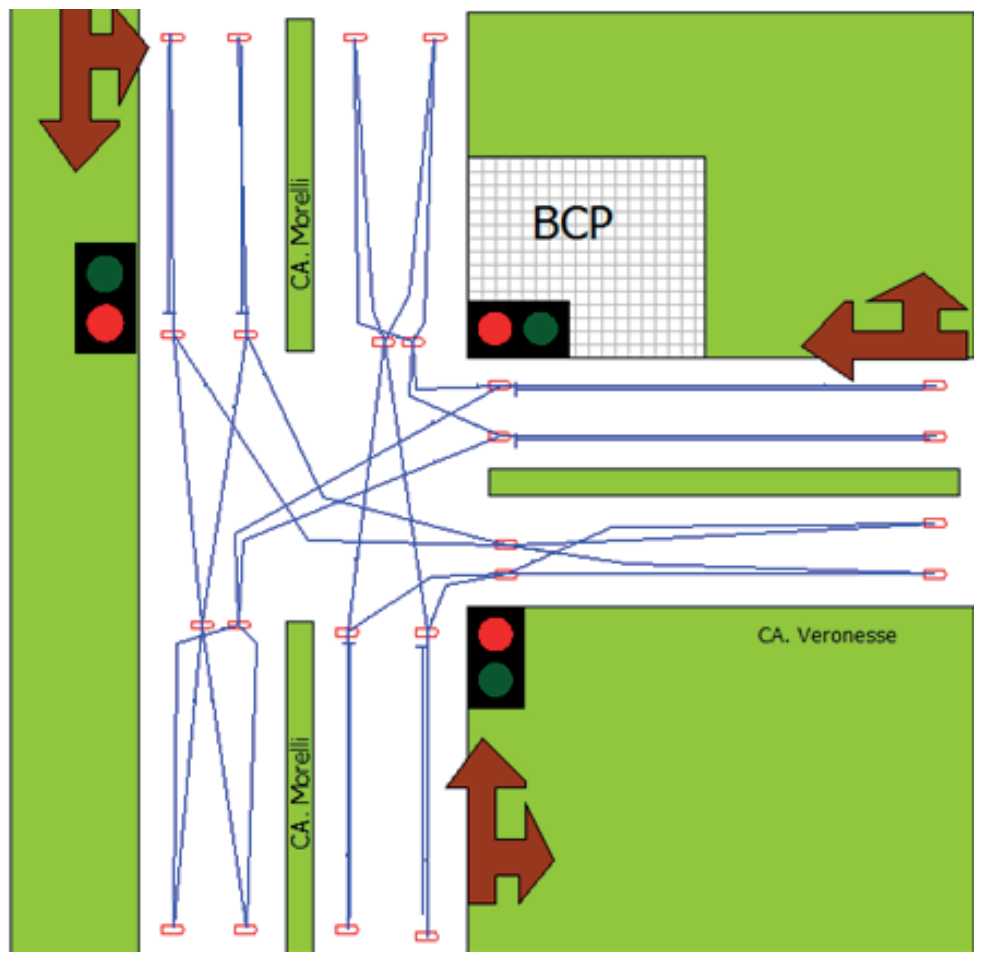

Figura 5. Animación Modelo en Arena

\begin{tabular}{|c|c|c|c|c|c|c|c|c|c|}
\hline Rangos & & & & & & & & & \\
\hline Media Real & 3,94 & 43,04 & 89,53 & 36,77 & 169,65 & 331,65 & 619,81 & 410,64 & 1531,75 \\
\hline Cant en el in & 8 & 9 & 8 & 10 & 8 & 10 & 10 & 9 & 10 \\
\hline$\%$ de confian & $80,00 \%$ & $90,00 \%$ & $80,00 \%$ & $100,00 \%$ & $80,00 \%$ & $100,00 \%$ & $100,00 \%$ & $90,00 \%$ & $100,00 \%$ \\
\hline Datos Reales & 4,00 & 44,00 & 82,00 & 45,00 & 172,00 & 327,00 & 619,00 & 408,00 & 1525,00 \\
\hline Cant en el in & 4 & 8 & 0 & 0 & 9 & 9 & 10 & 10 & 8 \\
\hline$\%$ de confian & $40,00 \%$ & $80,00 \%$ & $0,00 \%$ & $0,00 \%$ & $90,00 \%$ & $90,00 \%$ & $100,00 \%$ & $100,00 \%$ & $80,00 \%$ \\
\hline
\end{tabular}

Figura 6. Resultados de la Validación del Modelo para el caso T

\section{Conclusiones y Trabajos futuros}

En este trabajo se ha modelado y simulado usando Arena, 6 casos de transporte urbano para la ciudad de Lima, que es frecuente en las ciudades: Intersección, Ovalo, Unión, By-pass, Trébol y T, además se ha validado el modelo T.

En el modelaje, se observa que especificar la geometría de redes de transporte requiere una visita a campo, para conocer el ancho de calles, los posibles movimientos, las reglas de tránsito y la semaforización entre otros. Además, las variables que intervienen en cada modelo de red de transporte son muy parecidas y dependen del objetivo del estudio.
En la validación se consideró los flujos de transporte como procesos estocásticos que siguen una distribución Poisson de tasa variable, esto siguiendo las recomendaciones para el caso de transporte, y se obtuvo un 95\% de nivel de confianza.

\section{Trabajos Futuros}

Entre los trabajos futuros que se pueden derivar del presente estudio se encuentran: simular los casos de estudio mediante modelos en 3D, desarrollar un sistema para la obtención de datos flujo de transporte en tiempo real, e incluir nuevas variables como peatones y paraderos. 


\section{Agradecimientos}

Los autores agradecen a la secretaria técnica y la gerencia de transporte de Lima y Callao por facilitamos la información sobre estudios de tráfico de transporte urbano.

\section{Referencias}

[1] Instituto Metropolitano Protransporte de Lima, Estudio de Corredores Complementarios ALG - INOCSA -2006, http:// www.protransporte.gob.pe/biblio06.html, Setiembre 2014.

[2] K. Nellore, G. Hancke, A Survey on urban traffic management system using wireless sensor networks, Sensor(Basel). 2016 Jan 27; 16(2).

[3] Superintendencia Nacional de registros Públicos (SUNARP), Parque vehicular nacional estimado según clase de vehiculo: 1999 - 2008, http://www.mtc.gob.pe/estadisticas/update2/3.A.1.xls, Agosto 2012.

[4] Rosaldo J. F. Rossetti; Ronghui Liu, An Agent-Based Approach to Assess Drivers' Interaction with Pre-Trip Information Systems, Journal of Intelligent Transportation Systems, Volume 9, Issue 1 (2005) 1 - 10.

[5] Jerry Banks, Handbook of Simulation - Principles, Methodology, Advances, Applications and Practice, John Wiley \& Sons, 1998.

[6] J. Zambrano-Martinez, C. Calafate, D. Soler, J. Cano, P. Manzoni, Towards realistic urban traffic experiments using DFROUTER: Heuristic, validation and extensions, Sensors (Basel), 2017 Dec 15;17(12)

[7] David Kelton, Randall Sadowski, Nancy Zupick, Simulation with software Arena, Editorial McGraw-Hill, Sexta edición, 2015.

[8] Rockwell Automation, Arena Simulation Software, http://www. arenasimulation.com. Agosto 2011.
[9] Cal y Mayor y Asociados, S.C., Manual de Planeación y Diseño para la administración del tránsito y el transporte en Santa fe de Bogotá, BIRF 4021 A CO FONDATT-6, 2005.

[10] Christopher A. Chung, Simulation Modelling Handbook - A Practical Approach, CRC Press, 2004.

[11] Urban Traffic Control Systems, KonSULT, the Knowledgebase on Sustainable Urban Land use and Transport, http://www. elseviersocialsciences.com/transport/konsult/private/level2/ instruments/instrument014//2_014a.htm, Agosto 2010.

[12] Staffan Algers, Eric Bernauer, Marco Boero, Laurent Breheret, Carlo Di Taranto, Mark Dougherty, Ken Fox and JeanFrançois Gabard, SMARTEST Project Deliverable D3, European Commission under the Transport RTD Programme (1997) 23-29.

[13] Ronghui Liu and Shalini Sinha, Modelling Urban Bus Service and Passenger Reliability. The Third International Symposium on Transportation Network Reliability (INSTR) The Hague, the Netherlands 19th-20th July, (2007) 6-10.

[14] Z Mayaba, L de Beer and M Mokonyama, The Use of Dracula Microsimulation Modelling in the evaluation of public transport priority measures on the Soweto to parkdown corridor, Proceedings of $24^{\text {th }}$ Southern African Transport Conference (SATC), (2005) 1-10.

[15] J. Córdova, S, Campos, Modelos de Simulación de tráfico en redes de transporte urbano, caso: Lima, Perú, tesis de Ingeniería, UNMSM, 2012.

[16] Secretaria Técnica del Consejo de Transporte de Lima y Callao (CTLC). http://www.ctlc-st.gob.pe/index/home.htm, Agosto 2011.

[17] Gerencia de Transporte Urbano (GTU)- Sub Gerencia de Estudios de Transporte y Tránsito (SETT), agosto 2011. 
